\title{
Avaliação da resposta clínica ao uso de antibióticos por via oral e via inalatória em pacientes portadores de bronquiectasias
}

L. S. DE LIMA, M.BogOSSIAN

Trabalho desenvolvido pela Disciplina de Pneumologia da Universidade Federal de São Paulo - Escola Paulista de M edicina, São Paulo, SP.

RE SUMO - ОвJ етіvo. A base desta técnica foi obter alta concentração do antibiótico que excedia a concentração mínima inibitória (MIC) para a carga bacteriana presente no escarro.

Método. Para avaliar a resposta de pacientes portadores de bronquiectasias em quadros de exacer bações infecciosas ao tratamento com antibiótico por via oral (roxitromicina $300 \mathrm{mg} / \mathrm{dia}$ por 21 dias) e nos casos de fracasso deste esquema uso de antibiótico por via inalatória (gentamicina $80 \mathrm{mg} / 2$ vezes dia por 21 dias), foram avaliados em 28 pacientes atendidos no ambulatório da especialidade, alguns sinais e sintomas respiratórios de acordo com a escala de Cotes modi fi cada (secreção brônquica, tosse, broncoespasmo e dispnéia).

AnÁlise estatística. Utilizamos o seguinte tratamento estatísti co: teste de concordância Kappa e teste de McNemar para a discordância na avaliação dos graus de sinais e sintomas respiratórios, teste de Wilcoxon para os períodos sem infecções, teste exato de Fisher para os efeitos colaterais

\section{NTRODUÇÃO}

A bronquiectasia é uma doença caracterizada por dilatações anormais e definitivas de um ou mais brônquios, cujo quadro clínico clássico baseia-se na tríade: infecção respiratória recorrente, tosse crônica produtiva, hemoptise e/ou hemópticos ${ }^{1}$.

Trata-se de uma doença em declínio especialmente em países desenvolvidos, graças ao tratamento precoce e eficaz de infecções respiratórias, implementação de programas de reabilitação pulmonar e incrementação da imunização na infância, especialmente sarampo e coqueluche. Devemos consi derar que em nosso meio estas ações não se encontram no mesmo grau de desenvolvi mento, além de ressal tar o aumento do número de casos de tuberculose que poderá colaborar para uma maior incidência de bronquiectasia².

Diante do quadro que se configura nos países em desenvolvimento, a doença vem recebendo pouca atenção por parte de pesquisadores e profissionais de saúde em geral ${ }^{3}$, ficando reservado a esses apresentados, teste G de Cochran para análise dos antecedentes pessoais.

Resultados. Observamos que a presença dos antecedentes pessoais não influenciaram a evolução da infecção broncopulmonar, houve melhora significante nos sinais e sintomas avaliados excetuando-se a dispnéia que permaneceu inalterada em $80 \%$ dos casos.

Dıscussão. O grupo que fez uso de esquema antibiótico por via inalatória após fracasso do esquema via oral foi beneficiado com período médio sem infecção broncopulmonar significantemente superior, embora a presença de efeitos colaterais tenha sido superior neste grupo, porém sem repercussão clínica.

Conclusão. $O$ controle de quadros de exacerbação infecciosa em portadores de bronquiectasias foi melhor com o antibiótico por via inalatória do que por via oral.

UNITERMOS: Antibiótico. Inalação. Bronquiectasia.

pacientes o tratamento cirúrgico sob indicações criteriosas e normalmente considerado após um período prol ongado de fracasso da terapêutica conservadora (controle de secreções e antibioticoterapia).

A partir daí, estudos vem sendo desenvolvidos com o objetivo de avaliar novas estratégias de tratamento para a doença, especialmente no que se refere ao uso de antibióticos, já que a penetração de muitos na secreção purulenta endobrônquica é relativamente pobre e encontra condições desfavoráveis como baixo pH, presença de nucleoproteínas e alto conteúdo iônico ${ }^{4}$. A inativação enzimática dos antibióticos pelos patógenos presentes na secreção purulenta, bem como barreiras anatômicas anormais (fibrose, atelectasia, obliteração de vasos sanguíneos), dificultando a penetração local dos antibióticos, são outras limitações à obtenção de concentrações adequadas das drogas 5 .

Baseados nestes dados, resolvemos, estudar comparativamente o uso de antibióticos por via oral e inalatória com o objetivo de avaliar a evolução de 


\begin{tabular}{|c|c|c|c|c|}
\hline \multicolumn{5}{|l|}{ Após } \\
\hline \multicolumn{5}{|c|}{ 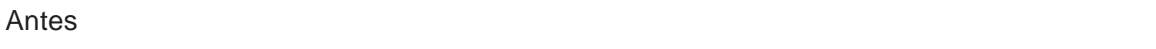 } \\
\hline Ausente & 0 & 0 & 0 & 0 \\
\hline Volume $<30 \mathrm{ml}$ & 0 & 0 & 0 & 0 \\
\hline Volume $>30 \mathrm{ml}$ & 2 & 7 & 7 & 16 \\
\hline TOTAL & 2 & 7 & 7 & 16 \\
\hline \multicolumn{5}{|c|}{$\begin{array}{l}\text { Teste de concordância Kappa } \\
z \text { calculado }=0,00 \quad z \text { crítico }=1,96 \\
\text { teste de } M c N e m a r \\
x^{2} \text { calculado }=9,00^{*} \quad x^{2} \text { crítico }=3,84 \\
\text { Concordância na diagonal }=43,7 \% \text { (inalterado) } \\
\text { Discordância acima da diagonal }=0,0 \% \text { (piorado) } \\
\text { Discordância abaixo da diagonal }=56,3 \% \text { (melhorado) }\end{array}$} \\
\hline
\end{tabular}

\begin{tabular}{|c|c|c|c|c|}
\hline $\begin{array}{l}\text { Após } \\
\text { Antes }\end{array}$ & AUSENTE & VOLUME $<30 \mathrm{ml}$ & VOLUME > 30ml & TOTAL \\
\hline Ausente & 0 & 0 & 0 & 0 \\
\hline Volume $<30 \mathrm{ml}$ & 0 & 0 & 0 & 0 \\
\hline Volume $>30 \mathrm{ml}$ & 0 & 5 & 7 & 12 \\
\hline TOTAL & 0 & 5 & 7 & 12 \\
\hline \multicolumn{5}{|c|}{$\begin{array}{l}\text { Teste de concordância Kappa } \\
z \text { calculado }=0,00 \quad \text { z crítico }=1,96 \\
\text { teste de } M c N e m a r \\
x^{2} \text { calculado }=5,00^{*} \quad x^{2} \text { crítico }=3,84 \\
\text { Concordância na diagonal }=58,3 \% \text { (inalterado) } \\
\text { Discordância acima da diagonal }=0,0 \% \text { (piorado) } \\
\text { Discordância abaixo da diagonal }=41,7 \% \text { (melhorado) }\end{array}$} \\
\hline
\end{tabular}

alguns sinais e sintomas respiratórios (tosse, dispnéia, broncoespasmo e secreção brônquica) nos esquemas antibióticos propostos.

\section{MÉTODO E CASUÍSTICA}

Foi realizado um estudo tipo antes/depois, com 28 pacientes, sendo 20 do sexo femi ni no com idade média de 52 anos (20 a 80 anos) e 8 pacientes do sexo masculino com idade média de 50,6 anos (19 a 74 anos). Estes pacientes eram portadores de bronquiectasias e assistidos no ambulatório desta especialidade da Disciplina de Pneumologia da UNIFESP-EPM. Os critérios de inclusão foram:

- Agudização dos sinais e sintomas respiratórios por infecção broncopulmonar.

- Tempo de agudização igual ou inferior a 3 semanas.

- Sem uso de antibiótico por igual período.

- Ausência de hemoptise e/ou hemópticos.

- Manutenção de medicamentos sintomáticos em uso.

- Creatinina sérica com valor normal.

Os pacientes foram divididos em dois grupos.
Inicialmente, todos os 28 pacientes receberam roxitromicina $300 \mathrm{mg}$ vo/dia, em dose única, durante 21 dias. Do total 16 responderam a esse esquema e constituiram o grupo I. Os 12 pacientes que continuaram com os sintomas e sinais inalterados, receberam gentamicina por via inalatória na dose de $80 \mathrm{mg} 2$ vezes no dia, constituiram o grupo II, entendendo-se como fracasso a manutenção dos sinais e sintomas iniciais.

Os pacientes receberam os inaladores, bem como as medicações e o material necessário. O inalador utilizado foi o modelo ultrassônico US800 da ICEL - Indústria e Comércio Eletrônica Ltda. F oram orientados quanto a ingestão do antibiótico (horári o e interval o al imentar) e ao preparo da inalação ( $10 \mathrm{ml}$ de soro fisiológico, 0,9\% e uma ampol a de gentamicina 80mg, inal ador ultrassônico).

O tempo previsto para a realização da inalação foi de 30 minutos, orientando os pacientes a realizar o procedimento enquanto a "névoa" estivesse saindo da máscara e manutenção da freqüência e "profundidade" habituais da respiração. Caso hou- 


\begin{tabular}{|c|c|c|c|c|}
\hline Após & AUSENTE & $\begin{array}{l}\text { ATIVIDADE } \\
\text { FÍSICA } \\
\text { PLENA }\end{array}$ & $\begin{array}{l}\text { ATIVIDADE } \\
\text { FÍSICA } \\
\text { LIMITADA }\end{array}$ & TOTAL \\
\hline \multicolumn{5}{|l|}{ Antes } \\
\hline Ausente & 1 & 0 & 0 & 1 \\
\hline Ativ. Física plena & 4 & 7 & 0 & 11 \\
\hline Ativ. Física limitada & 0 & 3 & 1 & 4 \\
\hline TOTAL & 5 & 10 & 1 & 16 \\
\hline \multicolumn{5}{|c|}{ 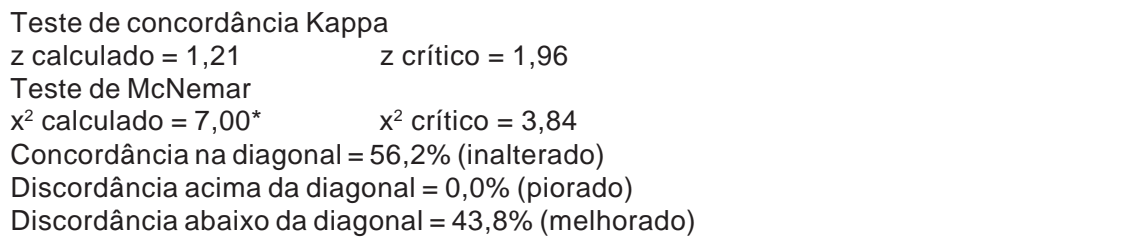 } \\
\hline
\end{tabular}

\begin{tabular}{|c|c|c|c|c|}
\hline Após & AUSENTE & $\begin{array}{l}\text { ATIVIDADE } \\
\text { FÍSICA } \\
\text { PLENA }\end{array}$ & $\begin{array}{l}\text { ATIVIDADE } \\
\text { FÍSICA } \\
\text { LIMITADA }\end{array}$ & TOTAL \\
\hline \multicolumn{5}{|l|}{ Antes } \\
\hline $\begin{array}{l}\text { Ausente } \\
\text { Ativ. Física plena } \\
\text { Ativ. Física limitada } \\
\text { TOTAL }\end{array}$ & $\begin{array}{l}1 \\
3 \\
0 \\
4\end{array}$ & $\begin{array}{l}0 \\
3 \\
3 \\
6\end{array}$ & $\begin{array}{l}0 \\
0 \\
2 \\
2\end{array}$ & $\begin{array}{r}1 \\
6 \\
5 \\
12\end{array}$ \\
\hline $\begin{array}{l}\text { Teste de concordânc } \\
z \text { calculado }=1,30 \\
\text { Teste de McNemar } \\
x^{2} \text { calculado }=6,00^{*} \\
\text { Concordância na dia } \\
\text { Discordância acima c } \\
\text { Discordância abaixo }\end{array}$ & $\begin{array}{l}\text { ppa } \\
z \text { crítico = } \\
\\
x^{2} \text { crítico } \\
=50,0 \% \text { (ina } \\
\text { agonal }=0,0 \% \\
\text { agonal }=50,0\end{array}$ & $\begin{array}{l}6 \\
84 \\
\text { ado) } \\
\text { orado) } \\
\text { melhorado) }\end{array}$ & & \\
\hline
\end{tabular}

vesse prescrição de uso de broncodilatador, este deveria preceder o antibiótico ${ }^{6}$.

Possíveis alterações na função renal foram monitorizadas através da dosagem de creatinina sérica ${ }^{6}$. Os sinais e sintomas respiratórios foram avaliados através da Escala de Cotes modificada, que é uma escala de classifi cação que correlaciona os graus de apresentação dos sinais e sintomas a valores numéri $\cos ^{8}$. Quanto a secreção brônquica, além da quantidade, avaliada pela escala, foi avaliado o aspecto (purulento e não purulento).

\section{Análise estatística}

- Teste G de Cochran para análise dos antecedentes pessoais ${ }^{8}$.

- Teste exato de Fisher para os efeitos colaterais apresentados 8 .

- Teste de Wilcoxon para os períodos sem infecções.

- Teste de Concordância Kappa e teste de McNemar para discordância na avaliação dos sinais e sintomas respiratórios?.
O nível de significância para rejei ção da hipótese de nulidade foi $\leq 5 \%$, assinalando-se com asterisco $(*)$ os valores significantes.

\section{RESULTADOS}

Os nossos achados estão inseridos nas Tabelas 1 a 13. Nas de número 1 a 8 estão escritos os val ores dos sinais e sintomas. A análise estatística segue as tabelas. $\mathrm{Na}$ Tabela 9 estão os valores dos períodos sem infecção nos pacientes que receberam o antibiótico por via oral e a seguir por via inalatória. Na tabela 10 o aspecto macroscópico do escarro antes e após os tratamentos e final mente na Tabela 11 os antecedentes pessoais e nas Tabelas 12 e 13 os efeitos colaterais.

\section{DISCUSSÃO}

Os resultados deste estudo mostraram que dos 28 pacientes em quadro de infecção broncopul- 


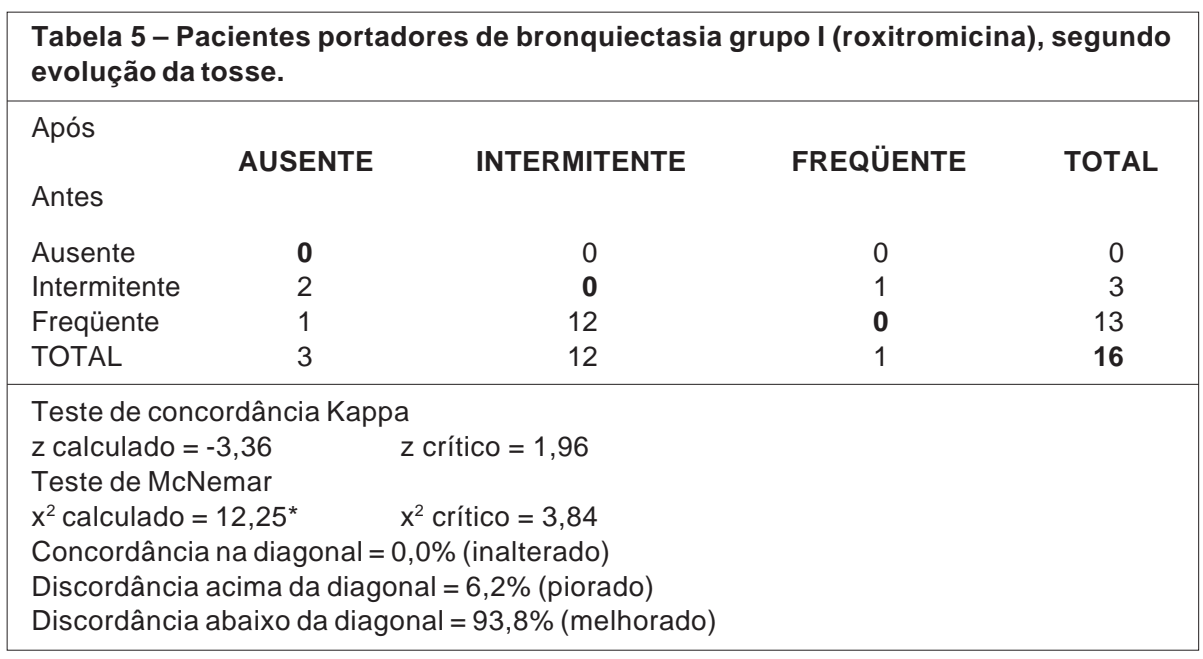

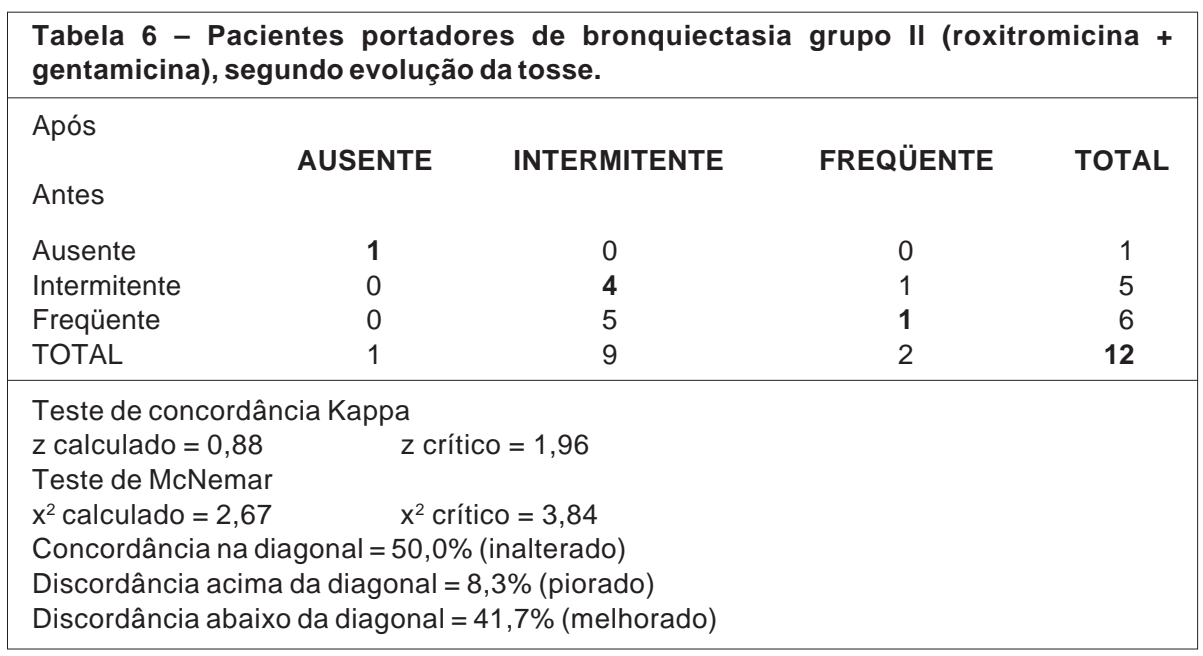

monar, tratados inicial mente com antibiótico por via oral, 16 deles (grupol) evoluíram com mel hora dos sinais e sintomas respiratórios avaliados (secreção brônquica, tosse, broncoespasmo, dispnéia) e 12 não obtiveram sucesso com o tratamento, permanecendo inalterados os sinais e sintomas avaliado, sendo então tratados em seguida com anti biótico por via inalatória e que vieram a constituir o grupo II.

A análise dos resultados mostra que em relação ao volume de secreção, ocorreu melhoria significante tanto no grupo I quanto no grupo II (Tabelas 1 e 2). Após o esquema do antibiótico por via inalatória $41,7 \%$ dos pacientes apresentaram diminuição do volume de secreção brônquica o que sem dúvida configurou uma vantagem para os pacientes que haviam fracassado com a antibióticoterapia por via oral.

Em relação ao broncoespasmo, ocorreu fato parecido, conforme análise das Tabelas 3 e 4, quanto à vantagem da via inalatória em $50 \%$ dos casos que fracassaram com a via oral. Quanto a tosse ocorreu o seguinte fato: no grupo I (Tabela 5) o esquema mostrou-se efi caz em $93,8 \%$ dos paci entes e apenas $6,2 \%$ tiveram o sintoma pi orado. Quanto ao grupo II (Tabela 6), embora a evolução estatística não tenha sido significante, ainda assim, 41,7\% dos casos melhoraram, 50\% permaneceram inalterados e $8,3 \%$ pioraram.

Já em relação à dispnéia a evolução não foi eficaz nem no grupo I (Tabela 7) e nem no grupo II (Tabela 8). Esse fato foi também constatado em 1981 por Wilson. Essa ocorrência poderia ser explicada por volumes el evados de secreção nas vias aéreas maior do que $30 \mathrm{ml}$. Em nossa casuística esse fato ocorreu em 7 pacientes $(58,3 \%)$ ou ainda pela terapia protussigena imposta ao grupo II pela inalação de solução sal ina mais anti biótico.

As reações adversas da terapia com anti bióti cos por via inalatória podem ser de três classes: nas vias aéreas, seleção de bactérias resistentes e efeitos tóxicos sistêmi cos da absorção da droga da luz do brônquio para o sangue (rim) ${ }^{5}$. 


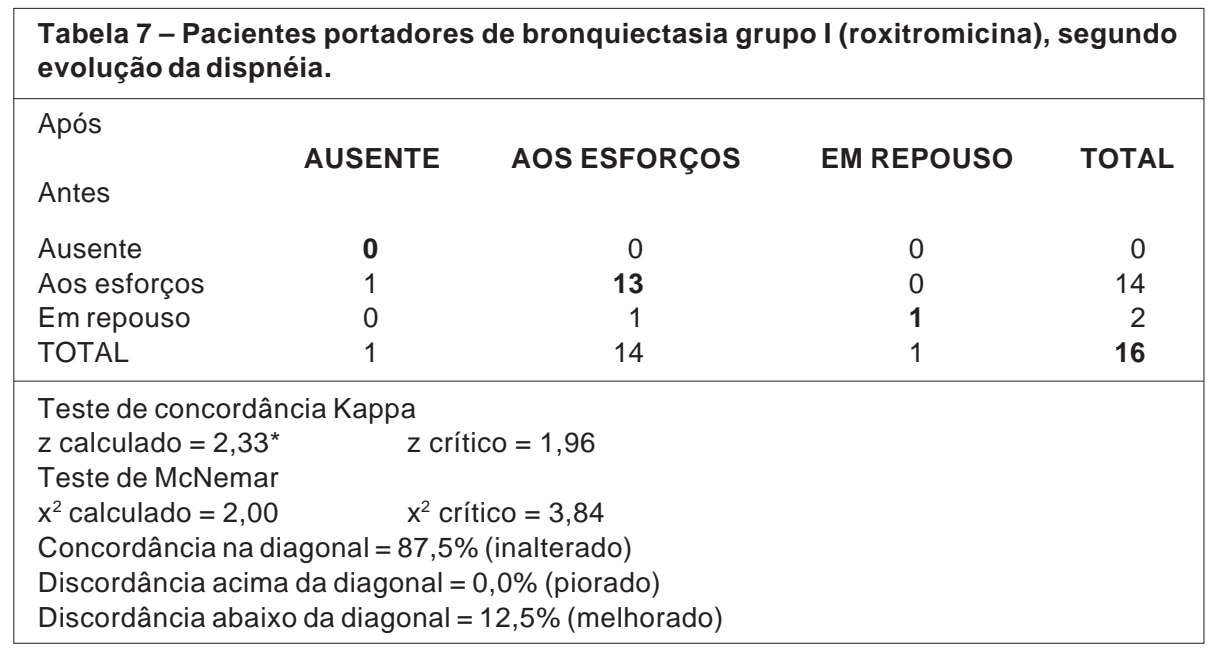

\begin{tabular}{|c|c|c|c|c|}
\hline $\begin{array}{l}\text { Após } \\
\text { Antes }\end{array}$ & AUSENTE & AOS ESFORÇOS & EM REPOUSO & TOTAL \\
\hline Ausente & 0 & 0 & 0 & 0 \\
\hline Aos esforços & 2 & 10 & 0 & 12 \\
\hline Em repouso & 0 & 0 & 0 & 0 \\
\hline TOTAL & 2 & 10 & 0 & 12 \\
\hline \multicolumn{5}{|c|}{$\begin{array}{l}\text { Não analisável } \\
\text { Concordância na diagonal = 83,3\% (inalterado) } \\
\text { Discordância acima da diagonal = 0,0\% (piorado) } \\
\text { Discordância abaixo da diagonal = 16,7\% (melhorado) }\end{array}$} \\
\hline
\end{tabular}

Pacientes que recebem antibiótico por via inalatória podem se queixar de opressão retroesternal, dispnéia e tosse. Não tivemos nenhum relato de opressão torácica; no grupo II como já descrito a evolução da tosse após o esquema inalatório não foi o desejado. Em relação à resposta da purulência após o uso dos dois esquemas de antibióticos (Tabela 10), verificamos que no grupo I todos os pacientes apresentavam purulência antes da roxitromicina e após o esquema um caso persistiu com escarro purulento, treze eram não- purulentos e dois pacientes não apresentavam mais expectoração. No grupo II, dez tornaram-se não purulentos e apenas dois persistiram com purulência. A evol ução foi boa com os antibióticos utilizados nos dois grupos, ressaltando o maior benefício no grupo II da via inalatória em relação ao esquema oral, provavelmente pela maior concentração alcançada nas secreções brônqui cas ou que essas bactérias não eram sensíveis ao antibiótico administrado por via oral.

Stockley, em 1987, descreve que o antibiótico administrado por via inalatória não agiria em rel ação à especifi ci dade e sensibilidade a determinada bactéria, e sim no "POOL" bacteriano. Os sintomas de irritação brônquica são muito mais freqüentes com o uso de polimixina e ticarcilina em portadores de broncopatia crônica e fibrose cística, respectivamente.

Do ponto de vista clínico o uso inalatório de gentamicina, carbenicilina, amoxilina, ceftazidima é bem tol erado cl inicamente, sendo relatados discretos rebaixamentos dos fluxos respiratórios, porém sem expressão clínica 5 . O objetivo racional do uso de antibióticos por via inalatória é obter concentração nas secreções brônquicas que exceda - MIC da bactéria. Por outro lado, em algumas situações como na bronquite crônica, as bactérias invadem a mucosa e portanto a concentração predominante do anti bióti co nas secreções brônquicas permanece controverso para essa situação. Alguns antibióticos tem maior penetração nas secreções e outros na mucosa brônquica 5 .

E m casos como tuberculose, mi coplasma e fungos, isto é, microrganismos intracelulares, a maior concentração do antibiótico nos macrófagos é hipótese atraente, promissora e em desenvol vimento, se atingindo essa meta através dos lipossomas ${ }^{5}$. Após instilação endo-traqueal ou através de inalação de 


\begin{tabular}{|c|c|c|}
\hline $\begin{array}{c}\text { Paciente } \\
\text { (Roxitromicina) }\end{array}$ & $\begin{array}{c}\text { Via Oral } \\
\text { (Gentamicina) }\end{array}$ & Via Inalatória \\
\hline 7 & 1,0 & 1,4 \\
\hline 9 & 3,0 & 4,3 \\
\hline 12 & 0,0 & 23,6 \\
\hline 13 & 0,0 & 8,9 \\
\hline 14 & 1,0 & 23,6 \\
\hline 15 & 3,9 & 4,3 \\
\hline 16 & 0,0 & 1,7 \\
\hline 17 & 1,0 & 8,3 \\
\hline 19 & 0,0 & 18,1 \\
\hline 21 & 0,0 & 0,0 \\
\hline 24 & 0,0 & 4,3 \\
\hline 28 & 3,6 & 10,0 \\
\hline Período médio & 1,13 & 9,04 \\
\hline \multicolumn{3}{|c|}{$\begin{array}{l}\text { Teste de Wilcoxon } \\
\text { t calculado }=0,0^{*} \\
\text { t crítico }=14,0 \\
\text { via oral }<\text { via inalatória }\end{array}$} \\
\hline
\end{tabular}

40mg de gentamicina à crianças com fibrose cística, traqueostomizados e com seqüelas neurológicas, a concentração do antibiótico na secreção brônquica foi respectivamente 700 e $55 \mu \mathrm{g} / \mathrm{ml}$ (Baran et al). Odio, em 1975, administrando $2 \mathrm{mg} / \mathrm{kg}$ a adultos traqueostomizadas obteve 43 e $14 \mu \mathrm{g} / \mathrm{ml}$ respectivamente 4 e 6 horas após a administração. Concentração el evada ocorreu aos 30 minutos - $480 \mu \mathrm{g} / \mathrm{ml}$.

O mesmo autor estudou a concentração da gentamicina em adultos não traqueostomizados, em amostras de secreção brônquica obtidas por aspiração trans-traqueal ou no escarro expectorado espontaneamente e em ambas as amostras a concentração foi $22 \mu \mathrm{g} / \mathrm{ml}$. A dose administrativa foi $2 \mathrm{mg} / \mathrm{kg} / \mathrm{peso}$. A maior concentração do anti bi ótico nas secreções funcionaria ou inibindo a adesão da Pseudomonas à mucosa ou a liberação de substâncias tóxicas pelas bactérias invasivas.

Estudos admitem, como maior efeito adverso para a administração de antibióticos inalatórios a seleção de cepas antibiótico-resistentes, porém estes trabal hos correlacionam esse fato com o uso profilático, ${ }^{11}$ sugerindo ser minimizado o problema com a alternância de drogas ${ }^{12,13}$. Thys, Aoun \& Klastersky, em 1994, descreveram esta resistência como rara e transitória, ocorrendo apenas após muitos meses de uso contínuo. A conseqüência clínica é controversa. Em outro estudo o índice de resistência foi el evado, porém sem prejuízo para o paciente. Os estudos demonstram cabal mente que nas secreções brônqui cas a concentração inibitória mínima para maioria ou para todas as bactérias é muito superior quando o anti bi ótico é admi nistra-

\begin{tabular}{|c|c|c|c|c|}
\hline \multicolumn{5}{|c|}{$\begin{array}{l}\text { Tabela } 10 \text { - Pacientes portadores de bronquiectasia do grupo } \\
\text { I (roxitromicina) e grupo II (roxitromicina + gentamicina), } \\
\text { segundo o aspecto da secreção brônquica). }\end{array}$} \\
\hline & \multicolumn{2}{|c|}{ GRUPO I } & \multicolumn{2}{|c|}{ GRUPO II } \\
\hline & ANTES & APós & ANTES & APós \\
\hline Ausente & 0 & 2 & 0 & 0 \\
\hline Purulento & 16 & 1 & 12 & 2 \\
\hline Não-purulento & 0 & 13 & 0 & 10 \\
\hline
\end{tabular}

Tabela 11-Pacientes portadores de bronquiectasia em relação ao percentual (\%) e número $(\mathrm{N})$ de antecedentes pes s o a is nos grupos I (roxitromicina) e grupo II (roxitromicina + gentamicina).

\begin{tabular}{|lcccc|}
\hline ANTECEDENTES & $\begin{array}{c}\text { Grupo I } \\
\text { N }\end{array}$ & $\begin{array}{c}\text { Grupo I } \\
\%\end{array}$ & $\begin{array}{c}\text { Grupo II } \\
\text { N }\end{array}$ & $\begin{array}{c}\text { Grupo II } \\
\%\end{array}$ \\
Tuberculose & 8 & 18,6 & 5 & 16,1 \\
Asma & 7 & 16,3 & 7 & 22,6 \\
Corpo estranho & 0 & 0,0 & 1 & 3,2 \\
Infecções repetição & 8 & 18,6 & 6 & 19,5 \\
Sinusite & 9 & 20,9 & 5 & 16,1 \\
Sarampo & 6 & 13,9 & 2 & 6,5 \\
Diabetes & 2 & 4,7 & 1 & 3,2 \\
Abscesso pulmonar & 0 & 0,0 & 1 & 3,2 \\
Coqueluche & 0 & 0,0 & 1 & 3,2 \\
Lobectomia & 3 & 7,0 & 1 & 3,2 \\
Cirurgia cisto nasal & 0 & 0,0 & 1 & 3,2 \\
Total & 43 & 100,0 & 31 & 100,0 \\
\hline Grupo I = Grupo II & \multicolumn{4}{|}{} \\
qui quadrado não analisável & \multicolumn{4}{|l}{} \\
\hline
\end{tabular}

do por via inalatória que por via sistêmica 5 .

O nosso trabalho mostrou que 12 pacientes $(42,8 \%)$ foram beneficiados quando fizeram uso de antibiótico inalatório apresentando o período sem infecção superior ao obtido com a técnica da via oral. As histórias destes pacientes trouxeram-nos relatos sobre doenças que antecederam ou estiveram associadas à doença atual. Conforme Tabela 11 observamos que a presença destes antecedentes foi significante dentro dos grupos I e II, embora o qui quadrado tenha sido não analisável intergrupos, portanto não sendo possível considerar a influência dos mesmo nos resultados obtidos com os esquemas de antibióticos.

A dosagem de creatinina sérica após o uso de antibiótico inalatório não apresentou nenhuma al teração importante, já que pacientes com prejuízo da função renal não foram incluídos no estudo. Os efeitos colaterais estiveram presentes em 2 casos do grupo I (12,5\%) e em 6 (50\%) dos pacientes do grupo II, destacando-se o aparecimento de epistaxe, especialmente em situações de sinusite ativa associada, porém nenhum dos efeitos col aterais motivou interrupção de tratamento (Tabela 12 e 13). Sabe-se que a absorção da gentami cina da secreção brônquica para o sangue é mai or quando 


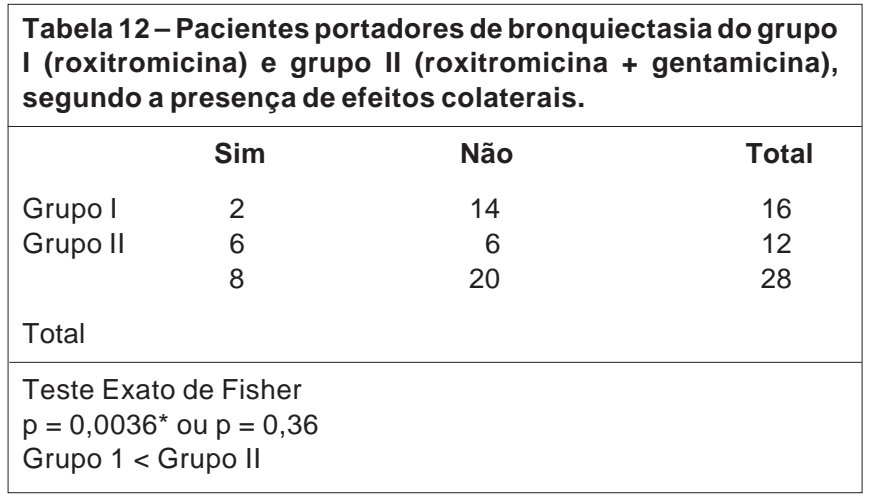

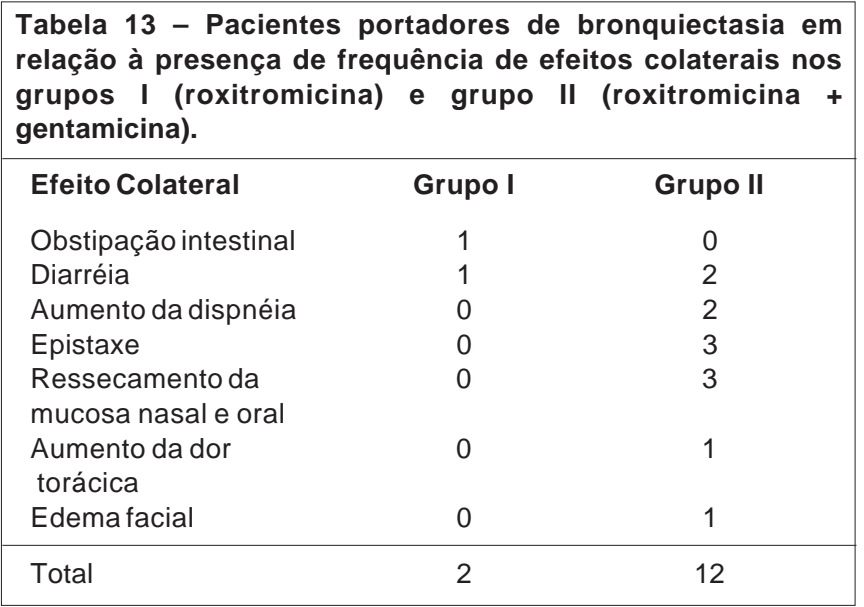

se instila a droga diretamente nas vias aéreas do que quando administrada por aerossol, recuperando-se na urina respectivamente, 15 a $17 \%$ e 1 a 5\% da dose total do antibiótico. Em indivíduos com função renal preservada o MIC sanguíneo excepcionalmente poderá ultrapassar $1 \mathrm{mg} / \mathrm{ml}$. Cuidado especial e monitoramento da creatinina é obrigatório em casos de insuficiência renal ${ }^{5}$.

Acreditamos que o uso de antibiótico inalatório não substitui o uso sistêmico dessa droga, e a necessidade das medidas de higiene brônquica no tratamento dos pacientes portadores de bronqui ectasias graves. Porém pode ser uma importante opção para um grupo de pacientes ambulatoriais, podendo-se evitar a internação hospitalar dos indivíduos com infecções crônicas recurrentes, diminuindo o impacto psicológico e menor ônus no tratamento desses pacientes.

O risco do aparecimento de cepas resistentes ao uso por longos períodos, de antibiótico por via inalatória, isso é profilático, por anos, quando necessário, pode ser atenuado com esquema intermitente. A tol erância para as vias aéreas é boa e os efeitos sistêmicos podem ser prevenidos.

Este trabalho teve o apoio financeiro CNPq. Parte da Tese de Doutorado de Luciane Soares de Lima, defendida e aprovada em 31/10/97 - Curso de Pós-Graduação em Ciências Pneumológicas (Disciplina de Pneumologia/ UNIFESP-EPM).

\section{SUMMARY}

\section{Evaluation of clinical response in the anti- biotics use by inhalatory and oral ways in patientes with bronchiectasis}

I NTRODUCTION. The aim of this technique was to achieve high concentration of the antibiotic that excedeed the minimal inhibitory concentration (MIC) of the microbial load present in the sputum.

MeTHOD. To evaluate the bronchiectasis patients with pictures of infectious exacerbations response to the treatment with antibiotic by oral way (roxitromicin $300 \mathrm{mg} /$ day for 21 days) and in cases of failure of this schema the use of antibiotic by inhal atory way (gentami cin $80 \mathrm{mg} / 2$ times day for 21 days), 28 patients were evaluated a special ambulatory, some signs and respi ratory symptoms according to the "Cotes modified scale" (sputum, cough, bronchospasm and dyspnea).

Statistical analysis. We used: Kappa concordant test and McNemar test for discordation in the evaluation of the degree of signal and respiratory symptoms, Wil coxon test for periods without infection, Fisher test for the collaterals effects presented, $G$ of Cochran test for the personal history analysis.

RESULTS. The personal history did not influence the evolution of bronchopulmonary infeccion, the evaluated signs and symptoms had significant improvement except dyspnea that stayed the same in $80 \%$ of the cases.

Discussion. The group that had used the antibi otics schema by inhal atory way after oral scheme failure had a significant longer period without bronchopulmonary infection but with superior collateral effects without clinicals repercussions.

CONCLUSION. The use of inhalatory antibiotic in infectious exacerbations in patients with bronchiectasis was better than the oral way. [Rev Ass Med Brasil 1999; 45(3):229-36.]

KEY WORDS: Antibiotics. Inhalation. Bronchiectasis

\section{REFERÊNCIAS BIBLIOGRÁFICAS}

1. Bogossian M, Romaldini H. Supurações Pulmonares. In: Prado FC, Ramos J A, ValleJ R, eds. AtualizaçãoTerapêutica. 18 ed. São Paulo, Artes Médicas, 1997; 134-39.

2. Bogossian M, Santoro IL, Jamnik S, Romaldini $H$. Bronquiectasia: Estudo de 314 casos. Tuberculose $X$ não tuberculose. J Pneumol 1998; 24 (1): 11-13.

3. Bardana EI, Barker AF. Brochiectasis. Uptade of na orphan disease. Am Rev Respir Dis 1988; 137:969-78. 
4. Wong GA, PeirceTH, Goldsteine, Hoeprich PD. Penetration of antimicrobial agents into bronchial secretions. Am J Med 1975; 59:219-23.

5. Thys J P, Aoun M, Klastersky J . Local antibiotic therapy for bronchopulmonary infections. I n: Remington J E. Respi ratory infections: Diagnosis and management. London, Raven Press, 1994; 741-65.

6. Glashan RQ. O que o enfermeiro deveria saber antes de administrar aminoglicosídeos ao cliente/paciente. Acta Paul Enf 1996; 9(1):45-52.

7. Cotes JE. Lung function: assesment and aplication in medicine. 2nd ed. Oxford, Blackwell Scientific Publication, $1968 ; 541$.

8. Siegel S, Castellan J r. NJ . Nonparametrics statistics. 2nd ed. Mc Graw-Hill. New York, 1988.

9. Landis J R, Koch GG. The measurement of observer agreement for contrasts among multinomial populations. Biometrics 1997; 33:159-74.

10. Wilson FE. Acute respiratory failure secondary to polymyxin B inhalation. Chest 1981; 79:237-39.
11. Levine BA, Petroff PA, Slado CL, Preutt BA. Prospective trials of dexamethasone and aerosolized gentamicin in the treatment of inhalation injury in theburned patient.J trauma 1978; 18:188-93.

12. Klick J M, Moulin GC, Hedley-Whyte J et al. Prevention of Gram-negative bacillary pneumonia using polymyxin aerosol as prophylaxis: Effect on the incidence of pneumonia in seri ously ill patients. J Clin Invest 1974; 55:514-19.

13. Klastersky J , Hensgens C, N oterman J . Endotracheal antibiotics for the prevention of trachobronchial infections in the tracheotomized unconsicions patients. Chest 1975; 68:302-6.

14. Baran D, Dachy A, Klastersky D. Concentration of gentamicin in bronchial secretions in children with cystic fibrosis or tracheostomy. Int Clin Pharmacol 1975; 12:336-41.

15. OdioW, Van Laer E, KlasterskyJ . Concentrations of gentamicin in bronchial secretions of intramuscular and endotracheal administration. Int Clin Pharmacol 1975; 15:518-24.

16. Stockley, R.A. - Bronquiectasis- new therapeutic approaches based on pathogenesis. Clin. Chest M ed, 1987, 83 (3): 481-494. 